\title{
Board / Committee Members
}

\section{Board of Society}

\begin{tabular}{|c|c|c|}
\hline Presid & Hiroyuki Nishikawa & $\begin{array}{r}\text { (Shibaura Institute of } \\
\text { Technology) }\end{array}$ \\
\hline \multirow{3}{*}{$\begin{array}{l}\text { Editor in Chief } \\
\text { Vice-President }\end{array}$} & Masayoshi Nagata & (University of Hyogo) \\
\hline & Katsuji Nakagawa & (Nihon University) \\
\hline & Hirotaka Muto (Mitsu & bishi Electric Corporation) \\
\hline \multirow{4}{*}{$\begin{array}{l}\text { Planning and } \\
\text { General Affairs } \\
\text { Treasurer }\end{array}$} & Yoshikazu Hoshina & (Toshiba Corporation) \\
\hline & Keisuke Yamashiro & (Fuji Electric Co., Ltd.) \\
\hline & Jumpei Kusukawa & , Ltd.) \\
\hline & Yasuyuki Kurata & Meidensha Corporation) \\
\hline \multirow[t]{2}{*}{ Editorial Affairs } & Mitsuaki Maeyama & (Saitama U \\
\hline & Akinori Oda (Chiba & Institute of $\mathrm{Te}$ \\
\hline \multirow[t]{3}{*}{$R \& D$ Management } & Keizo Kato & (Niigata University) \\
\hline & Toshihiro Takahashi & (Central \\
\hline & & Electri \\
\hline \multirow[t]{2}{*}{ Auditor } & Masayuki Hikita & $\begin{array}{r}\text { (Kyushu Institute of } \\
\text { Technology) }\end{array}$ \\
\hline & Ko & (Iwate University) \\
\hline \multirow[t]{3}{*}{ Member } & Yukitaka Shinoda & (Nihon University) \\
\hline & $\begin{array}{l}\text { Hiroaki Miyake } \\
\text { Haruo Ihori }\end{array}$ & $\begin{array}{r}\text { (Tokyo City University) } \\
\text { (Ehime University) }\end{array}$ \\
\hline & & $\begin{array}{r}\text { (Kyushu Institute of } \\
\text { Technology) }\end{array}$ \\
\hline
\end{tabular}

\section{R\&D Steering Committee}

$\begin{array}{llr}\text { Chairperson } & \text { Katsuji Nakagawa } & \text { (Nihon University) } \\ \text { Vice-Chairperson } & \begin{array}{l}\text { Keizo Kato } \\ \text { Toshihiro Takahashi }\end{array} & \text { (Niigata University) } \\ & \begin{array}{l}\text { (Central Research } \\ \text { Institute of Electric Power Industry) }\end{array}\end{array}$

Member

$$
\text { Akihito Otani (Nihon University) }
$$

Masashi Ohchi (Chiba Institute of Technology) Mitsuhiro Kusaba (Osaka Sangyo University) Akiko Kumada (The University of Tokyo) Keiji Goto (National Defense Academy) Koichi Takaki (Iwate University) Naoki Hayakawa (Nagoya University) Kunihiko Hidaka (The University of Tokyo) Masafumi Yashima (Tohoku University) Masahiro Yamaguchi (Tohoku University) Kenichi Yamazaki (Central Research Institute of Electric Power Industry)

Nobuyuki Yoshikawa (Yokohama National University)

\section{Editorial Committee}

Chairperson Hirotaka Muto (Mitsubishi Electric Corporation) Vice-Chairperson Mitsuaki Maeyama (Saitama University) Akinori Oda (Chiba Institute of Technology) Member Masashi Ohchi (Chiba Institute of Technology) Naoyuki Shimomura (Tokushima University) Hisashi Miyazaki (National Defense Academy) Daigo Yonetsu (Kansai University) Shizue Furukawa (Central Research Institute of Electric Power Industry)

Masaki Nakano (Nagasaki University) Yohei Ishikawa (National Institute of Technology, Ariake College) Ryosuke Ozaki (Nihon University)
Masateru Ikehata

(Railway Technical

Research Institute)

Susumu Suzuki (Chiba Institute of Technology) Takeshi Kinoshita (Keio University) Seitaro Kon (National Institute of Advanced Industrial Science and Technology)

Hiroshi Morita (Hitachi, Ltd.) Satoru Kuboya (Toshiba Energy Systems \& Solutions Corporation)

Makoto Sonehara (Shinshu University) Toshiyuki Sawa (Hitachi, Ltd.)

Mitsuaki Maeyama (Saitama University) Secretary Takahiro Imai (Toshiba Energy Systems \& $\begin{array}{rr}\text { Yutaka Noguchi } & \text { (Meliji University) }\end{array}$

\section{Editorial Board}

Editor in Chief Masayoshi Nagata (University of Hyogo) Assistant Editor Yusuke Kikuchi (University of Hyogo)

[Group A1: Common Interests]

Co-Editor in Chief Masashi Ohchi (Chiba Institute of Technology) Associate Editor Daigo Yonetsu (Kansai University) Yukitaka Shinoda (Nihon University) Toshihiko Shibazaki (Tokyo Metropolitan College of Industrial Technology)

Kenji Takahara (Fukuoka Institute of Technology) Hisaya Tanaka (Kogakuin University)

27 members

[Group A2: Fundamentals]

Co-Editor in Chief Naoyuki Shimomura (Tokushima University) Associate Editor Shizue Furukawa (Central Research Institute of Electric Power Industry)

Satoshi Ihara Naomichi Ezumi (University of Tsukuba) Douyan Wang (Kumamoto University) Yasunori Ohtsu (Saga University) Hiroki Kojima (Nagoya University) Takashi Yagisawa (Toshiba Memory Corporation) 126 members

[Group A3: Materials]

Co-Editor in Chief Hisashi Miyazaki (National Defense Academy) Associate Editor Masaki Nakano (Nagasaki University) Mitsuyoshi Onoda (University of Hyogo) Muneaki Kurimoto (Nagoya University) Masahiro Kozako (Kyushu Institute of Technology)

Makoto Sonehara (Shinshu University) Kazuyuki Tohyama (National Institute of Technology, Numazu College) Norikazu Fuse (Central Research Institute of Electric Power Industry) Hiroi Yamaguchi (JFE Steel Corporation) 79 members

(Updated on June $1^{\text {st }}, 2018$ ) 